\title{
Einleitung: \\ Berufsbildungs- und Hochschulpolitik in der Schweiz, Österreich und Deutschland
}

\author{
Christine Trampusch und Marius R. Busemeyer \\ Universität Bern und Max-Planck-Institut für Gesellschaftsforschung, Köln
}

Politikwissenschaftliche Bildungsforschung hat bislang vier Schwerpunkte: Vergleichende Policy-Analyse, Prozesse der Internationalisierung und Europäisierung, Arbeiten der vergleichenden politischen Ökonomie sowie Studien zu den Effekten von Bildungsinstitutionen und -politiken. Auf der Grundlage einer dichten Analyse der gegenwärtigen Transformation und Reform von Bildungs- und Ausbildungssystemen in der Schweiz, Österreich und Deutschland ergänzen die Artikel- und Forumsbeiträge dieses Sonderheftes diese Perspektiven um drei spezifische Punkte, die in der zukünftigen Forschung zum Wandel von Bildungssystemen vertieft werden sollten: erstens die Untersuchung der Wechselwirkungen zwischen Bildungspolitik und der föderalen Entscheidungsarena, zweitens die Analyse des Einflusses von Machtverteilung und der Dynamik politischer Koalitionen auf Reformen und drittens die Unterscheidung zwischen Reformpolitik und Prozessen des institutionellen Wandels.

KeYwords: Higher Education • Vocational Training $\bullet$ Switzerland $\bullet$ Austria $\bullet$ Germany

\section{Einleitung ${ }^{1}$}

Bildungs- und Berufsbildungssysteme sind zentrale Institutionen politischer Ökonomien, weil sie sowohl für die Wirtschaft als auch für die Politik von enormer Bedeutung sind. Für die Leistungsfähigkeit nationaler, regionaler und lokaler Ökonomien gelten Bildung und Ausbildung als ein wichtiger Erfolgsfaktor. Bildungs- und Berufsbildungssysteme gehören aber auch

\footnotetext{
1 Wir danken der gesamten Redaktion der Swiss Political Science Review und insbesondere Matthias Meyer-Schwarzenberger und Christine Scheidegger für die professionelle Betreuung dieses Sonderheftes. Unser besonderer Dank gilt ferner den Gutachterinnen und Gutachtern der Artikelbeiträge dieses Sonderheftes.
} 
zum Kern nationalstaatlicher Identitäten. Sie sind das Ergebnis nationaler, politischer, wirtschaftlicher und sozialer Entwicklungen und Interessenauseinandersetzungen, nicht beliebig austauschbar und daher sehr stabil. Diese Argumente gelten in besonderem Maße für die Bildungssysteme der Schweiz, Deutschlands und Österreichs. Starke föderalistische Staatsstrukturen, die Mitwirkung der Sozialpartner vor allem in der beruflichen Bildung und, im Fall der Schweiz, die Bedeutung direktdemokratischer Instrumente machen groß angelegte Bildungsreformen unwahrscheinlich.

In den letzten Jahren jedoch hat die Transformation und Reform von Bildungs- und Ausbildungssystemen nicht nur in der europäischen und internationalen Politik, sondern auch zunehmend in der Schweiz, Deutschland und Österreich an Dynamik gewonnen. Schlagwörter und Symptome wie Hochschulreformen, Lehrstellenkrise, die abnehmende Ausbildungsbereitschaft der Betriebe und die Anpassung von Qualifikationen an die Erfordernisse des ökonomischen Strukturwandels prägen nationale Debatten. Auch auf EU-Ebene nehmen Bildung und Ausbildung im Rahmen des Lissabon-Prozesses einen hohen Stellenwert ein. Mit dem BolognaProzess und dem Kopenhagen-Prozess hat die EU in der Hochschul- und Berufsbildungspolitik damit begonnen, einen Europäischen Bildungsraum zu schaffen und durch die Einführung eines Europäischen Qualifikationsrahmens Systeme der beruflichen Bildung miteinander vergleichbarer zu machen.

Ziel dieses Sonderheftes ist es, durch national vertiefende und vergleichend angelegte Analysen zu Institutionen und Akteuren der Bildungspolitik ein umfassendes und systematisches Bild der Ursachen, Muster und Folgen der gegenwärtigen Transformation und Reform von Bildungs- und Ausbildungssystemen in der Schweiz, Deutschland und Österreich zu zeichnen. Hierbei wird ein besonderer Schwerpunkt auf den post-sekundären Bildungsbereich gelegt, d.h. berufliche Bildung, Hochschulbildung und Weiterbildung. Der Primar- und Sekundarschulsektor wird in den Fallstudien weniger intensiv untersucht. Diese Schwerpunktsetzung liegt darin begründet, dass zum Einen besonders in den Bereichen der beruflichen und der hochschulischen Bildung besagte Transformationen zu beobachten sind. Zum Anderen ist die Schulpolitik in föderalistisch organisierten Ländern wie den hier betrachteten stark durch regionale und lokale Gegebenheiten geprägt, so dass der relative Mehrgewinn von Ländervergleichen auf der Aggregatebene geringer ausfällt.

Das Sonderheft gliedert sich in zwei Teile: Der erste Teil enthält fünf Studien zur Berufs- und Hochschulbildung in der Schweiz, Österreich und 
Deutschland, wobei der Schweizer Fall ausführlicher diskutiert wird als der Fall Deutschland und vor allem Österreich. ${ }^{2}$ Thematisch sprechen die Artikelbeiträge dieses Sonderheftes drei Oberthemen an: Berufsbildung und Hochschulbildung als Politikfeld, Bildungspolitik im Föderalismus und Berufs- und Hochschulbildung in der EU. Der zweite Teil des Sonderheftes enthält in Form von Kurzbeiträgen Kommentare von Forschern und Praktikern zu aktuellen Bildungsreformen sowie Präsentationen weiter gehender Forschungsprojekte im Feld der sozialwissenschaftlichen Bildungsforschung. Zusammen genommen zeigen die Forschungsartikel sowie die Beiträge zum Forum, dass, erstens, die Bildungslandschaften der vermeintlich reformresistenten Länder Schweiz, Deutschland und Österreich in den letzten Jahren in Bewegung geraten sind. Zweitens wird deutlich, dass die Politikwissenschaft diese Entwicklung nachvollzieht und sich zunehmend eine politikwissenschaftliche Perspektive auf die Bildungsforschung etabliert. Es ist Ziel dieses Sonderheftes, diese Perspektive weiter zu entwickeln.

Um einen kurzen Überblick über diese Einleitung zu geben: Im ersten Abschnitt stellen wir kurz die politikwissenschaftliche Literatur zur Berufs- und Hochschulbildung vor, wobei wir vor allem auf die internationalen Debatten eingehen. Daran anschließend führen wir in die Beiträge des Sonderheftes ein und stellen kurz die wichtigsten Ergebnisse vor. Abschließend ziehen wir ein Fazit, in dem wir auf weiterhin offene Fragen und Perspektiven künftiger Forschung eingehen.

\section{Überblick über politikwissenschaftliche Bildungsforschung}

Nachdem die Bildungsforschung von der Politikwissenschaft lange Zeit vernachlässigt wurde, hat das Interesse an diesem Politikbereich - vor allem in der internationalen Politikwissenschaft - in den letzten Jahren immens zugenommen (vgl. auch die Übersichten zum Forschungsstand in Busemeyer und Trampusch 2011a, b, both forthcoming). Wenn man die vergleichende Forschung stärker in den Blick nimmt und dabei auch Studien an der Grenze zur Bildungssoziologie, Bildungsökonomie sowie den Erziehungswissenschaften einbezieht, so können vier Themenkomplexe

\footnotetext{
2 Dies ist eine Konsequenz der ungleichen Verteilung der eingereichten Vorschläge für dieses Sonderheft. Bedauerlicherweise kann der Fall Österreich in diesem Sonderheft nur in dem vergleichenden Beitrag von Christian Ebner und Rita Nikolai zur Berufsbildung thematisiert werden.
} 
identifiziert werden: vergleichende Bildungsforschung im Stile der vergleichenden Policy-Forschung, die Analyse der zunehmenden Internationalisierung und Europäisierung von Bildungspolitik, vergleichende politökonomische Analysen von Bildungspolitik sowie die Untersuchung der Effekte von Bildungspolitik und -systemen (vgl. dazu ausführlich Busemeyer und Trampusch 2011a, forthcoming).

Die vergleichende Policy-Analyse (comparative public policy) befasst sich mit den Fragen, "wie, warum und in welchem Ausmaß" (Heidenheimer, Heclo und Tech Adams 1990: 3) Regierungen, politische Parteien und sozioökonomische Akteure unterschiedliche bildungspolitische Strategien verfolgen. Zurückgehend auf die frühen Arbeiten von Heidenheimer (1981, 1997), Windolf (1990) und Archer (1984) wird untersucht, wie politische Institutionen, das relative Timing makrosozialer Prozesse wie Demokratisierung und Industrialisierung sowie die Verteilung von Machtressourcen zwischen gesellschaftlichen Klassen und sozialen Bewegungen die unterschiedlichen Entwicklungspfade von Bildungssystemen geprägt haben. Beiträge in dieser Forschungstradition zielten vor allem auf die dichte Beschreibung von historischen Entwicklungen und waren nur in Teilen an die etablierten Theorieansätze der vergleichenden Policy-Forschung angeschlossen. Außerdem wurde der Bereich der beruflichen Bildung zunächst ausgeblendet. Diese Forschungslücke hat Kathleen Thelen (2004, 2007; Culpepper und Thelen 2008) mit ihrer Studie zur Entstehung der beruflichen Bildungssysteme Deutschlands, Japans, Großbritanniens und der USA geschlossen. In neueren Studien in der Tradition der vergleichenden Staatstätigkeitsforschung steht die Analyse der Bildungsfinanzen im Vordergrund (Busemeyer 2007, 2008, 2009c; Castles 1989; Jensen 2011, forthcoming; Nikolai 2007; Schmidt 2003, 2004, 2007; Wolf 2006; Wolf und Zohlnhöfer 2009).

Der zweite Themenkomplex politikwissenschaftlicher Bildungsforschung untersucht den Prozess der Internationalisierung und Europäisierung von Bildungspolitik. Nicht nur die EU (Dobbins und Knill 2009; Martens und Wolf 2006; Powell und Trampusch 2011, forthcoming; Trampusch 2008, 2009), auch die OECD (Jakobi und Martens 2007), die WTO und GATS (Osterwalder und Weber 2004; Scherrer 2007) werden zunehmend zu bedeutenden Akteuren in der Bildungspolitik, zumal die Internationalisierung der Wirtschaft Prozesse der Deregulierung und Privatisierung von Bildungsinstitutionen befördert. Auf internationaler Ebene hat sich somit ein neues Politikfeld etabliert, in dem vorrangig weiche, auf Koordination basierende Verfahren (Benchmarking, die Methode der of- 
fenen Koordinierung) Anwendung finden, die in den nationalen Bildungssystemen aber durchaus nachhaltige Transformationsprozesse in Gang setzen können. Dies wird nicht zuletzt durch Fallstudien in diesem Sonderheft bestätigt (vgl. den Beitrag von Bieber). Eine Erklärung für diesen scheinbar paradoxen Befund ist in der Logik von Mehrebenenspielen zu finden. Nationale Akteure befördern die Europäisierung der Bildungspolitik, weil sie damit darauf abzielen, nationale Reformblockaden zu überwinden (siehe Busemeyer 2009b, d; Trampusch 2008, 2009 für den Bereich der Berufsbildung sowie Martens und Wolf 2006; Witte 2006 für das Feld der Hochschulbildung).

Drittens hat die politikwissenschaftliche Bildungsforschung durch Arbeiten der vergleichenden politischen Ökonomie, vor allem den "Varietiesof-Capitalism"-Ansatz, erheblich an Bedeutung gewonnen. Peter Hall und David Soskice (2001) weisen auf die Bedeutung der beruflichen Bildung in der Ausprägung spezifischer Wettbewerbsvorteile in der Produktion von Hochqualitätsgütern in koordinierten Markwirtschaften hin (vgl. auch zuvor schon Streeck 1989, 1992). Estévez-Abe, Iversen und Soskice (2001) diskutieren den Zusammenhang zwischen Humankapitalinvestitionen und Sozialpolitik und zeigen, dass ein ausgebautes System der beruflichen Bildung mit einer geringeren sozialen Ungleichheit einhergeht. Iversen und Stephens (2008) verfolgen diesen Gedanken weiter und arbeiten strukturelle Ähnlichkeiten und gemeinsame politische Ursprünge zwischen Bildungsregimen und Esping-Andersens (1990) "Welten des Wohlfahrtskapitalismus" heraus. Die Analyseperspektive der vergleichenden politischen Ökonomie und des "Varieties of Capitalism"-Ansatzes wird insbesondere auf die Berufsbildungssysteme der Schweiz, Österreichs und Deutschlands angewandt. Eine Reihe von Studien verweisen darauf, dass für den Wandel in diesen Systemen - historisch wie auch aktuell - die Konstellation im Arbeitgeberlager und Koalitionen, die die Arbeitgeber mit staatlichen Eliten bilden, entscheidend sind (Busemeyer 2009a, d; Culpepper 2007; Rohrer und Trampusch 2011, forthcoming; Thelen 2004; Trampusch 2010a,b,c).

Viertens gibt es eine Fülle von Studien aus der Bildungssoziologie und -ökonomie, die die Effekte und Auswirkungen von Bildungsinstitutionen und -politiken auf Outcomes untersuchen. Im Unterschied zu dem ersten hier vorgestellten Forschungsfeld geht es also nicht primär darum, Unterschiede im Policy Output zu erklären, sondern welche realen sozialen und ökonomischen Veränderungen Policy Output erzeugt. Erst in jüngerer Zeit hat dieses Thema auch das Interesse von Politikwissenschaftlern gefunden (Freitag und Schlicht 2009; Freitag, Schlicht und Stadelmann-Steffen 
2010), obwohl es von elementarem Interesse ist, die Rückwirkung von bildungspolitischen Outputs und Outcomes auf politische Entscheidungsprozesse zu verstehen ("policies determine politics", Lowi 1972: 299).

So weit die kurze Zusammenfassung der internationalen Debatten in der politikwissenschaftlichen Bildungsforschung. Wenngleich hier ein Aufwärtstrend im Interesse deutlich zu erkennen ist, liegen umfassende politikwissenschaftliche Monographien zur Hochschul- und Berufsbildungspolitik in der Schweiz, Österreich und Deutschland nur in sehr begrenztem Maße vor. Es dominieren Arbeiten aus der Bildungssoziologie, der Bildungsökonomie und den Erziehungswissenschaften (vgl. den Überblick in Tippelt und Schmidt 2009). Natürlich gibt es einige Ausnahmen von dieser Regel, zum Beispiel (und ohne Anspruch auf Vollständigkeit) für das Feld der Berufsbildung: Offe (1975), Kübler (1986), Rüegg (1986), Streeck et al. (1987), Gonon (1998), Gonon und Kiener (1998), Culpepper und Finegold (1999), Culpepper (2003), Rothe (2001), Thelen (2004) und Busemeyer (2009d); und für die Hochschul- und allgemeine Bildungspolitik: Windolf (1990), Heidenheimer (1997), Wolf (2006), von Below (2002) und Kreft (2006). Es kommt hinzu, dass in den letzten Jahren eine Reihe von politikwissenschaftlichen Studien in Fachzeitschriften erschienen sind, die deutlich zeigen, dass die Hochschul- und Ausbildungssysteme in den drei Ländern zunehmend zu einem zentralen Forschungsgegenstand der Politikwissenschaft geworden sind. Beispiele dafür stellen die folgenden Studien dar: Busemeyer 2009a b; Culpepper 2007; Freitag und Schlicht 2009; Martens und Wolf 2006; Sager 2006; Trampusch 2008, 2009, 2010a,b,c.

Dieses Sonderheft möchte an dieser Stelle ansetzen und einen Beitrag dazu leisten, die politikwissenschaftliche Erschließung der Bildungssysteme der Schweiz, Deutschlands und Österreichs zu befördern. Angesichts des stark wachsenden Interesses am Themenfeld sowie der prominenten Rolle, die diesen Ländern in einigen der international geführten Debatten wie etwa der "Varieties-of-Capitalism"-Diskussion zukommt, erscheint dies dringend geboten. Zum Zweiten sind mittlerweile in vielen europäischen Ländern signifikante Veränderungen in den Systemen der beruflichen Bildung und dem Hochschulsystem zu erkennen, deren Antriebskräfte und Auswirkung noch nicht verstanden sind. Im Folgenden möchten wir zeigen, wie die einzelnen Beiträge dieses Sonderheftes zur Ausprägung eines besseren Verständnisses der skizzierten Entwicklungen beitragen. Dies geschieht entlang der bereits angesprochenen drei Oberthemen. 


\section{Berufsbildung und Hochschulbildung als Politikfeld}

Die ersten drei Beiträge dieses Sonderheftes analysieren Berufsbildung und Hochschulbildung aus der Perspektive der Politikfeld-Analyse. Christian Ebner und Rita Nikolai untersuchen in vergleichender Perspektive Unterschiede innerhalb der kollektiven Ausbildungsregime in Deutschland, Österreich und der Schweiz. Die Berufsbildungssysteme dieser drei Länder werden oftmals als ähnlich wahrgenommen. Ebner und Nikolai zeigen jedoch einige wichtige Unterschiede auf. Ein besonders gewichtiger Unterschied besteht darin, dass sich in Österreich neben dem dualen System der Berufsausbildung ein starker und wachsender Zweig der vollzeitschulischen Berufsbildung etabliert hat. Auf der Grundlage ihrer vergleichenden Analyse kommen Ebner und Nikolai zu dem Ergebnis, dass drei zentrale Erklärungsfaktoren diesen Unterschied erklären können: Vollzeitschulische Ausbildung kann sich dann parallel neben der dualen Ausbildung entwickeln, wenn erstens Gewerkschaften und Arbeitgeber dies unterstützen, sich zweitens eine informelle Große Koalition zwischen Sozialdemokraten und Christdemokraten für den Ausbau der vollzeitschulischen Berufsausbildung einsetzt und drittens der Zentralstaat - und nicht die Gliedstaaten - die Gesetzgebungskompetenzen im Bereich der vollzeitschulischen Berufsbildung innehat. Der Beitrag zeigt dementsprechend auf, dass weniger die Unternehmen allein - wie im "Varieties-ofCapitalism"-Ansatz angenommen - oder sozio-ökonomische Faktoren die länderspezifische Mischung aus dualer und vollzeitschulischer Berufsausbildung erklären. Vielmehr arbeiten die Autoren heraus, dass die politicsDimension und damit die Dynamik von Koalitionen politischer Akteure innerhalb und jenseits des Regierungssystems im engeren Sinne die zentralen Determinanten sind. Dies stellt eine wichtige Ergänzung zu arbeitgeberzentrierten Studien des "Varieties-of-Capitalism"-Ansatzes dar.

Der Beitrag von Ruth Kamm und Michaela Köller ist explorativ ausgerichtet und untersucht aus der Governance-Perspektive der Hochschulforschung den Wandel der Hochschulsysteme in den 16 deutschen Bundesländern. Wie in anderen Ländern sind auch die deutschen Hochschulen einem starken Reformdruck ausgesetzt. In Anlehnung an das Konzept des New Public Management sollen diese Reformen zu einer Steigerung der Steuerungsautonomie und damit der Effizienz des Systems als Ganzes beitragen. Auch wenn die öffentliche Debatte über diese Themen sehr aktuell ist, gibt es noch keine umfassende empirische Studie, die das Ausmaß dieses Wandels empirisch und über die einzelnen Bundesländer verglei- 
chend untersucht. Kamm und Köller schließen diese Forschungslücke. Sie unterscheiden zwei Governance-Modelle: das bürokratisch-oligarchische Modell, einerseits, und das Management-Modell, anderseits. Es folgt eine systematische Inhaltsanalyse der Landeshochschulgesetze, die den Stand der Umsetzung des Management-Modells auf Bundesländerebene aufzeigt. Kamm und Köller finden heraus, dass die Hochschulen in Hessen und Nordrhein-Westfalen am meisten dem Management-Modell folgen, während sich Berlin und Rheinland-Pfalz weiterhin durch ihre Nähe zum bürokratisch-oligarchischen Modell auszeichnen. Der Beitrag stellt des Weiteren fest, dass es keinen systematischen Zusammenhang zwischen dem Abschneiden der Hochschulen in der Exzellenzinitiative und dem Grad der Umsetzung des Management-Modells gibt.

Unter Anwendung des Konzepts der Pfadabhängigkeit sowie den kapital- und feldtheoretischen Überlegungen zu Hochschulen von Bourdieu untersucht der Artikel von Karl Weber, Patricia Tremel und Andreas Balthasar den Aufbau der Fachhochschulen in der Schweiz. Diese wurden in einem "unschweizerisch rasanten Tempo" Mitte der 1990er Jahre eingeführt. Die Autoren zeigen, dass es vor allem die Akteure der Berufsbildung waren, die den Aufbau der Fachhochschulen geprägt haben. Dies hat zur Folge, dass sich die Fachhochschulen weitgehend unabhängig von den universitären Strukturen in der Schweiz entwickeln. Weil der Prozess des Aufbaus der Fachhochschulen in diesem Sinne pfadabhängig verlief, unterscheiden sich die Fachhochschulen bezüglich ihrer Organisation, des wissenschaftlichen Personals, der Eingangsvoraussetzungen für Studierenden und den Bedingungen in der Lehre heute auch deutlich von den Universitäten.

\section{Bildungspolitik im Föderalismus}

Die Beiträge von Thomas Griessen und Dietmar Braun sowie von Manuel Fischer, Pascal Sciarini und Denise Traber untersuchen die Wechselwirkung zwischen Bildungspolitik und Föderalismus und bieten dabei unterschiedliche, sich in der Gesamtheit ergänzende Perspektiven an. Griessen und Braun widmen ihren Beitrag der Frage, welche Auswirkungen die Bildungsreformen der letzten Jahre, die in Deutschland und der Schweiz umgesetzt wurden, auf die Gestaltung der föderalen Entscheidungsarena haben. Fischer, Sciarini und Traber hingegen untersuchen, wie die spezifische Ausgestaltung der politischen Institutionen der Schweiz den Ent- 
scheidungsprozess zur Einführung der neuen Verfassungsartikel zur Bildungspolitik geprägt haben.

Thomas Griessen und Dietmar Braun betrachten vergleichend föderale Koordinations- und Verflechtungsstrukturen zwischen Bund und Ländern bzw. Kantonen in der Hochschulpolitik Deutschlands und der Schweiz und zeigen einen erstaunlichen Unterschied auf: Obwohl die deutsche Föderalismusreform auf eine Entflechtung der Kompetenzen gerade auch im Bildungsbereich zielte, argumentieren die Autoren, dass trotz der Reformen weiterhin ein hohes Maß an Politikverflechtung herrscht. Im Gegensatz dazu zielten die Schweizer Reformen auf eine Stärkung der Koordination in der Bildungspolitik. Damit sei die Schweizer Hochschulpolitik heute im Vergleich zu früher wesentlich stärker verflochten. Dabei haben sich jedoch Mechanismen entwickelt, die zukünftige Entscheidungsblockaden möglicherweise verhindern können. Obwohl sich damit die GovernanceStrukturen in der Hochschulpolitik der beiden Länder angenähert haben, können die Autoren zeigen, dass die Gefahr einer Politikverflechtung in der Schweiz geringer ist als in Deutschland, das trotz der Bemühungen um Entflechtung weiterhin mit der Politikverflechtungsfalle zu kämpfen hat.

Auf der Basis von Eliteninterviews und einer dichten Analyse von Primärdokumenten und quantitativen Daten untersucht der Beitrag von Manuel Fischer, Pascal Sciarini und Denise Traber aus der Perspektive des Politikverflechtungskonzepts und unter Anwendung der Methode des "analytical narratives" schließlich den Entscheidungsprozess zur Einführung der neuen Bildungsverfassungsartikel in der Schweiz. Die Reform, die im Mai 2006 von Volk und Ständen gutgeheissen wurde, stärkt die Rolle der Eidgenossenschaft gegenüber den Kantonen. Dies war nicht unbedingt zu erwarten, da die Schweizer Bildungspolitik sehr dezentralisiert ist und durch die Veto-Möglichkeiten der Kantone das Zuschnappen der Politikverflechtungsfalle drohte. Die Autoren zeigen, dass vor allem die auf Problemlösung ausgerichtete Interaktionsorientierung der Akteure diese Reform ermöglichte. Insbesondere identifizieren sie die folgenden vier Faktoren, die halfen, die Politikverflechtungsfalle zu überwinden: die Herausbildung gemeinsamer Überzeugungen und Einschätzungen, die Trennung von verfassungspolitischen und distributiven Reformthemen, die Präsenz eines neutralen Brokers und das Vorhandensein informeller Strukturen. 


\section{Berufs- und Hochschulbildung in der EU}

Der Beitrag von Tonia Bieber zur Europäisierung der Schweizer Hochschulund Berufsbildungspolitik rundet die Artikelbeiträge dieses Sonderheftes schließlich durch eine Berücksichtigung internationaler Einflüsse ab. Dass die Schweiz, obwohl sie nicht Mitglied der EU ist, massiven Einflüssen der EU unterliegt, ist dort in vielen Politikfeldern zu beobachten. Bieber zeigt in ihrem Beitrag, dass auch die Schweizer Bildungspolitik nicht von Europäisierung verschont ist. Hinsichtlich des Bologna-Prozesses gilt die Schweiz als eine Vorreiterin. Jedoch zeigen sich weit weniger Effekte in der Berufsbildung. Die Literatur zur Europäisierung und zur Konvergenz von Politiken kombinierend und auf der Grundlage einer dichten prozessanalytischen Rekonstruktion der Schweizer Reformen, bietet uns Bieber die folgende Lösung für dieses Puzzll an: Während in der Hochschulpolitik eine innenpolitische Reformkoalition zwischen Bildungsverwaltung, Universitäten und Universitätsorganisationen den Bologna-Impuls für eine Strukturreform des Hochschulwesens nutzte, verhinderten innerstaatliche Veto-Akteure, dabei insbesondere der Schweizerische Gewerbeverband (SGV), eine Europäisierung der Berufsbildungspolitik. Die Autorin bestätigt damit nicht nur bereits bestehende Studien zur Mehrebenendynamik des Bologna-Prozesses (z.B. Martens und Wolf 2006), sondern auch solche zur wichtigen Rolle der Wirtschaftsverbände in der Europäisierung der Schweiz (Mach 2006; Trampusch 2010c). Bieber ergänzt diese Literatur jedoch damit, dass sie auf die Bedeutung von transnationalen Kommunikationsprozessen verweist.

\section{Forum "Bildungsreformen und Forschungsprojekte"}

An die längeren Beiträge schließen sich im Rahmen eines Diskussionsforums mehrere Kurzbeiträge zu aktuellen Bildungsreformen und weiter gehenden Forschungsprojekten der sozialwissenschaftlichen Bildungsforschung an. Beat Hotz-Hart greift das Thema des Beitrags von Weber und Tremel wieder auf und diskutiert die Auswirkungen der Gründungen der Fachhochschulen auf das Schweizer Hochschulsystem. Stefan Denzler und Stefan C. Wolter präsentieren erste Befunde aus einem Forschungsprojekt, das untersucht, inwiefern die Entfernung zwischen Heimatort und Universität Studienentscheidungen beeinflusst. Benedetto Lepori und Tatiana Fumasoli berichten von einem Projekt aus der angewandten Bildungsfor- 
schung, das den Zusammenhang zwischen Bildungsreformen und Systemperformanz ausleuchtet. Ebenfalls mit dem Fall Schweiz befasst sich der Kurzbeitrag von Lucien Criblez, Christina Huber und Lukas Lehmann, die in einem Projekt die Reform der Lehrerbildung untersucht haben. Volker Rein reflektiert und kommentiert den Entscheidungsprozess, der zur Etablierung des Deutschen Qualifikationsrahmens (DQR) geführt hat. Abschließend diskutiert Barbara Dickhaus die möglichen Auswirkungen der Liberalisierung des Handels mit Dienstleistungen auf den Bildungssektor.

\section{Fazit}

Die Beiträge in diesem Sonderheft belegen die Vielfältigkeit und das Potential vergleichender, politikwissenschaftlicher Bildungsforschung, zeigen aber auch lohnende Felder für zukünftige Forschungsvorhaben auf. Wir möchten an dieser Stelle drei Punkte weiter ausführen.

Der erste Punkt betrifft die Wechselbeziehungen zwischen Bildungspolitik und der Ausgestaltung politischer Institutionen, insbesondere der föderalistischen Entscheidungsarena. Prima facie wäre zu erwarten, dass die ausgebauten föderalistischen Strukturen in den betrachteten Ländern Bildungsreformen erheblich erschweren. Sicherlich trifft es auch zu, dass Reformprozesse in den konsensual-korporatistischen Demokratien der Schweiz, Deutschlands und Österreichs langsamer verlaufen als in Mehrheitsdemokratien. Dennoch belegen die Beiträge dieses Sonderhefts, dass in den letzten Jahren beachtliche Transformationen stattgefunden haben - und zwar trotz des starken Föderalismus. Fischer, Sciarini und Traber zeigen, wie im Schweizer Fall die drohende Politikverflechtungsfalle vermieden werden konnte. Es bleibt zu untersuchen, ob bzw. wie dies auch in den anderen Ländern gelingen konnte. Der Beitrag von Griessen und Braun wirft außerdem die Frage der Rückwirkungen von Bildungsreformen auf die Gestaltung politischer Institutionen auf. Hier wäre von Interesse zu erfahren, inwiefern sub-nationale Akteure das Instrument der Bildungsreformen tatsächlich strategisch einsetzen wollen, um eine Transformation der politischen Arena zu ihren Gunsten zu erreichen, und ob diese nationalen Mehrebenen-Spiele mit Prozessen der Internationalisierung und Europäisierung der Bildungspolitik interagieren (vgl. den Beitrag von Bieber).

Zweitens zeigen die Beiträge von Ebner und Nikolai sowie Fischer, Sciarini und Traber, dass neben Institutionen die Dimension der politics 
eine wichtige Rolle spielt, d.h. die Machtverteilung zwischen gesellschaftlichen Kräften und die Dynamik politischer Koalitionen. Wie Kathleen Thelen (2004) in ihren Arbeiten zur Berufsbildungspolitik gezeigt hat, betrifft dies nicht unbedingt Regierungskoalitionen im engeren Sinne, sondern formelle und informelle Koalitionen politischer und ökonomischer Akteure, die nicht nur den policy output an sich beeinflussen, sondern auch institutionellen Wandel von Bildungssystemen jenseits gesetzgeberischer Maßnahmen befördern können. Der Beitrag von Ebner und Nikolai verweist allerdings auch auf die Bedeutung kritischer Weichenstellungen, die von Regierungsparteien bewusst vorgenommen werden. Bislang fehlt in der Literatur jedoch eine systematische Integration der Perspektive der klassischen Parteiendifferenzthese (Schmidt 1996) mit den neueren Ansätzen der historisch orientierten vergleichenden politischen Ökonomie (Thelen 2004).

Schließlich ist, drittens, deutlich geworden, dass die Beziehung zwischen policy output in Form von Bildungsreformen auf der einen Seite und dem tatsächlich zu beobachtenden institutionellen Wandel auf der anderen Seite noch in weiten Teilen unklar ist. Anknüpfend an die lange Tradition der Implementationsforschung geht der Beitrag von Kamm und Köller erste Schritte in diese Richtung. Im Lichte neuerer Ansätze zu Theorien des institutionellen Wandels (Streeck und Thelen 2005; Mahoney und Thelen 2010) sollte der Mismatch zwischen dem angestrebten Politikziel und dem tatsächlichen Outcome jedoch weniger als Versagen eines umfassenden Steuerungsanspruchs verstanden werden, wie es in der klassischen Implementationsforschung tendenziell der Fall war. Es ist vielmehr davon auszugehen, dass die selektive Umsetzung und gleichzeitige Transformation bestehenden Normen und Regelungen selbst als politischer, durch Machtfragen bestimmter Prozess zu verstehen ist, der dabei wiederum die Grundlagen zukünftiger politischer Entscheidungen zu beeinflussen sucht. Die Bildungspolitik bietet sich in besonderer Weise als Gegenstand der Analyse dieser wechselseitigen Interaktion zwischen policies und politics an, denn aufgrund des dezentralen Charakters dieser institutionellen Regime, sei es in der beruflichen Bildung oder der Hochschulbildung, kommt es regelmäßig zu "Steuerungsproblemen", d.h. dem Auftreten von systematischen Gaps zwischen Reformanspruch und -ergebnis. Ein besseres Verständnis der politischen und institutionellen Entstehungsbedingungen dieser Lücken wäre nicht nur von akademischem Interesse, sondern hat auch eine hohe Relevanz für die praktische Bildungspolitik. 


\section{Literatur}

Archer, M. (1984). Social Origins of Educational Systems. London: Sage. Busemeyer, M. (2007). The Determinants of Public Education Spending in 21 OECD Democracies, 1980-2001. Journal of European Public Policy 14(4): 582-610.

(2008). The Impact of Fiscal Decentralization on Education and Other Types of of Spending. Swiss Political Science Review 14(3): $451-81$.

(2009a). Die Sozialpartner und der Wandel in der Politik der beruflichen Bildung seit 1970. Industrielle Beziehungen 16(3): 273-94.

(2009b). Europäisierung der deutschen Berufsbildungspolitik. Aus Politik und Zeitgeschichte 45/2009: 25-31.

(2009c). Social Democrats and the New Partisan Politics of Public Investment in Education. Journal of European Public Policy 16(1): $107-26$.

- (2009d). Wandel trotz Reformstau: Die Politik der beruflichen Bildung seit 1970. Frankfurt: Campus.

Busemeyer, M. und C. Trampusch (2011a, forthcoming). Comparative Political Science and the Study of Education. British Journal of Political Science (forthcoming).

(Hrsg.), (2011b, forthcoming). The Comparative Political Economy of Collective Skill Formation Systems. Oxford: Oxford University Press (forthcoming).

Castles, F. (1989). Explaining Public Education Expenditure in OECD Nations. European Journal of Political Research 17(4): 431-48.

Culpepper, P. (2003). Creating Cooperation: How States Develop Human Capital in Europe. Ithaca, NY: Cornell University Press.

- (2007). Small States and Skill Specificity: Austria, Switzerland and Interemployer Cleavages in Coordinated Capitalism. Comparative Political Studies 40(6): 611-37.

Culpepper, P. und D. Finegold (Hrsg.), (1999). The German Skills Machine: Sustaining Comparative Advantage in a Global Economy. New York: Berghahn Books.

Culpepper, P. und K. Thelen (2008). Institutions and Collective Actors in the Provision of Training: Historical and Cross-National Comparisons. In Mayer, K. und H. Solga (Hrsg.), Skill Formation: Interdisciplinary and Cross-National Perspectives. Cambridge: Cambridge University Press (21-49). 
Dobbins, M. und C. Knill (2009). Higher Education Policies in Central and Eastern Europe. Convergence Toward a Common Model? Governance 22(3): 397-430.

Esping-Andersen, G. (1990). The Three Worlds of Welfare Capitalism. Cambridge: Polity Press.

Estévez-Abe, M., Iversen, T. und D. Soskice (2001). Social Protection and the Formation of Skills: A Reinterpretation of the Welfare State. In Hall, P. und D. Soskice (Hrsg.), Varieties of Capitalism: The Institutional Foundations of Comparative Advantage. Oxford: Oxford University Press (145-83).

Freitag, M. und R. Schlicht (2009). Educational Federalism in Germany: Foundations of Social Inequality in Education. Governance 22(1): 47-72.

Freitag, M., Schlicht, R. und I. Stadelmann-Steffen (2010). Educational Inequality in the EU: The Effectiveness of the National Education Policy. European Union Politics 11(1): 29-59.

Gonon, P. (1998). Das internationale Argument in der Berufsbildungsreform. Die Rolle internationaler Bezüge in den bildungspolitischen Debatten zur schweizerischen Berufsbildung und zur englischen Reform der Sekundarstufe II. Bern: Peter Lang.

Hall, P. und D. Soskice (2001). An Introduction to Varieties of Capitalism. In Hall, P. und D. Soskice (Hrsg.), Varieties of Capitalism: The Institutional Foundations of Comparative Advantage. Oxford: Oxford University Press (1-68).

Heidenheimer, A. (1981). Education and Social Security Entitlements in Europe and America. In Flora, P. und A. Heidenheimer (Hrsg.), The Development of Welfare States in Europe and America. New Brunswick, London: Transaction Books (269-304).

Heidenheimer, A. (1997). Disparate Ladders: Why School and University Policies Differ in Germany, Japan, and Switzerland. New Brunswick, London: Transaction Publishers.

Heidenheimer, A., Heclo, H. und C. Teich Adams (1990). Comparative Public Policy: The Politics of Social Choice in America, Europe, and Japan. 3rd edition. New York: St. Martin's Press.

Iversen, T. und D. Stephens (2008). Partisan Politics, the Welfare State, and Three Worlds of Human Capital Formation. Comparative Political Studies 41(4-5): 600-37.

Jakobi, A. und K. Martens (200). Diffusion durch Internationale Organisationen: Die Bildungspolitik der OECD. In Holzinger, K., Jörgens 
H. und C. Knill (Hrsg.), Politische Vierteljahresschrift, Sonderheft: Transfer, Diffusion und Konvergenz von Politiken 38: 247-70.

Jensen, C. (2011, forthcoming). Capitalist Systems, De-industrialization, and the Politics of Public Education. Comparative Political Studies 44 (forthcoming).

Kiener, U. und P. Gonon (1998). Die Berufsmatur als Fallbeispiel schweizerischer Berufsbildungspolitik. Umsetzungsbericht. Nationale Forschungsprogramm 33 Wirksamkeit unser Bildungssysteme. Aarau: SKBF.

Kreft, J. (2006). Gewerkschaften und Spitzenverbände der Wirtschaft als bildungspolitische Akteure: Positionen, Strategien und Allianzen. Wiesbaden: VS Verlag für Sozialwissenschaften.

Kübler, M. (1986). Berufsbildung in der Schweiz: 100 Jahre Bundessubventionen (1884-1984). Bern: Bundesamt für Industrie, Gewerbe und Arbeit.

Lowi, T. (1972). Four Systems of Policy, Politics and Choice. Public Administration Review 32(4): 298-309.

Mach, A. (2006). La Suisse entre internationalisation et changements politiques internes: législation sur les cartels et relations industrielles dans les années 1990. Zürich: Rüegger.

Mahoney, J. und K. Thelen (2010). Chapter 1. A Theory of Gradual Institutional Change. In Mahoney, J. und K. Thelen (Hrsg.), Explaining Institutional Change: Ambiguity, Agency, and Power. New York: Cambridge University Press (1-37).

Martens, K. und K. Wolf (2006). Paradoxien der Neuen Staatsräson: Die Internationalisierung der Bildungspolitik in der EU und der OECD. Zeitschrift für Internationale Beziehungen 13(2): 145-76.

Nikolai, R. (2007). Sozialpolitik auf Kosten der Bildung? Verteilungskonkurrenz in Zeiten knapper Kassen. Zeitschrift für Sozialreform 53(1): 7-30.

Offe, C. (1975). Berufsbildungsreform: Eine Fallstudie über Reformpolitik. Frankfurt: Suhrkamp.

Osterwalder, F. und K. Weber (2004). Die Internationalisierung der föderalistischen Bildungspolitik. Schweizerische Zeitschrift für Bildungswissenschaften 26(1): 11-32.

Powell, J. und C. Trampusch (2011, forthcoming). Europeanization of Collective Skill Formation Systems. In Busemeyer, M. und C. Trampusch (Hrsg.), (2011, forthcoming), The Comparative Politi- 
cal Economy of Collective Skill Formation Systems. Oxford: Oxford University Press (forthcoming).

Rohrer, L. und C. Trampusch (2011, forthcoming). Continuity and Change in the Swiss Vocational Training System. In Trampusch, C. und A. Mach (Hrsg.), Switzerland in Europe, Europe in Switzerland. Continuity and Change in the Swiss Political Economy. London: Routledge (forthcoming).

Rothe, G. (2001). Die Systeme beruflicher Qualifizierung Deutschlands, Österreichs und der Schweiz im Vergleich: Kompendium zur Ausund Weiterbildung unter Einschluss der Problematik lebensbegleitendes Lernen. Villingen-Schwenningen: Neckar-Verlag.

Rüegg, E. (1986). Neokorporatismus in der Schweizerischen Berufsbildungspolitik. Kleine Studien zur Politischen Wissenschaft Nr. 250. Zürich.

Sager, F. (2006). Erfolgsfaktoren von Lehrstellenmarketing in der dualen Berufsbildung: Das Beispiel Schweiz. International Review of Education 52(5): 461-78.

Scherrer, C. (2007). GATS: Commodifying Education via Trade Treaties. In Martens, K., Rusconi, A. und K. Leuze (Hrsg.), New Arenas of Education Governance: The Impact of International Organizations and Markets on Educational Policy Making. Basingstoke: Palgrave Macmillan (117-35).

Schmidt, M. (1996). When Parties Matter: A Review of the Possibilities and Limits of Partisan Influence on Public Policy. European Journal of Political Research 30(2): 155-83.

Schmidt, M. (2003). Ausgaben für Bildung im internationalen Vergleich. Aus Politik und Zeitgeschichte B21-22: 6-11. (2004). Die öffentlichen und privaten Bildungsausgaben Deutschlands im internationalen Vergleich. Zeitschrift für Staats- und Europawissenschaften 2(1): 7-31.

(2007). Testing the Retrenchment Hypothesis: Educational Spending, 1960-2002. In Castles, F. (Hrsg.), The Disappearing State? Retrenchment Realities in an Age of Globalisation. Cheltenham, Northampton: Edward Elgar (159-83).

Streeck, W. (1989). Skills and the Limits of Neo-Liberalism: The Enterprise of the Future as a Place of Learning. Work, Employment and Society 3(1): 89-104.

(1992). On the Institutional Conditions of Diversified Quality Production. In Streeck, W. und E. Matzner (Hrsg.), Beyond Keynesian- 
ism: The Socio-Economics of Production and Full Employment. Aldershot, Brookfield et al.: Edward Elgar (21-61).

Streeck, W., Hilbert, J., v. Kevelaer, K.-H. und F. Maier (1987). Steuerung und Regulierung der beruflichen Bildung: Die Rolle der Sozialpartner in der Ausbildung und beruflichen Weiterbildung in der Bundesrepublik Deutschland. Berlin: edition sigma.

Streeck, W. und K. Thelen (2005). Introduction: Institutional change in advanced political economies. In Streeck W. und K. Thelen (Hrsg.), Beyond Continuity: Institutional Change in Advanced Political Economies. Oxford: Oxford University Press (1-39).

Thelen, K. (2004). How Institutions Evolve: The Political Economy of Skills in Germany, Britain, the United States and Japan. Cambridge: Cambridge University Press.

(2007). Skill Formation and Training. In Jones, G. und J. Zeitlin (Hrsg.), The Oxford Handbook of Business History. Oxford: Oxford University Press (558-80).

Tippelt, R. und B. Schmidt (Hrsg.), (2009). Handbuch Bildungsforschung. Wiesbaden: VS Verlag für Sozialwissenschaften.

Trampusch, C. (2008): Jenseits von Anpassungsdruck und Lernen: Die EU-Berufsbildungspolitik und die Europäisierung der deutschen Berufsbildungspolitik. Zeitschrift für Staats- und Europawissenschaften 6(4): 577-605.

(2009). Europeanization and Institutional Change in Vocational Education and Training in Germany and Austria. Governance 22(3): 371-97.

(2010a). Co-evolution of Skills and Welfare in Coordinated Market Economies? A Comparative Historical Analysis of Denmark, the Netherlands, and Switzerland. European Journal of Industrial Relations 16(3): 197-220.

(2010b). Employers, the State, and the Politics of Institutional Change. Vocational Education and Training in Austria, Germany and Switzerland. European Journal of Political Research 49(4): $545-73$.

(2010c). The Politics of Institutional Change. Transformative and Self-Preserving Change in the Vocational Education and Training System in Switzerland. Comparative Politics 42(2): 187-206.

Von Below, S. (2002). Bildungssysteme und soziale Ungleichheit: Das Beispiel der neuen Bundesländer. Opladen: Leske und Budrich. 
Windolf, P. (1990). Die Expansion der Universitäten 1870-1985: Ein internationaler Vergleich, Stuttgart: Ferdinand Enke Verlag.

Witte, J. (2006). Change of Degrees and Degrees of Change. Comparing Adoptions of European Higher Education Systems in the Context of the Bologna Process. University of Twente, Netherlands. Twente.

Wolf, F. (2006). Die Bildungsausgaben der Bundesländer im Vergleich: Welche Faktoren erklären ihre beträchtliche Variation? Münster: LIT.

Wolf, F. und R. Zohlnhöfer (2009). Investing in Human Capital? The Determinants of Private Education Expenditure in 26 OECD Countries. Journal of European Social Policy 19(3): 230-44.

\section{Introduction: \\ Policies of Vocational Training and Higher Education in Switzerland, Austria, Germany}

Research on education from the perspective of political science has so far been concentrated in four areas: comparative public policy analysis, internationalization and Europeanization of education systems, work in the tradition of comparative political economy and studies on the effect of education policies and institutions. On the basis of an in-depth analyses of reforms and transformations of education and training systems in Switzerland, Austria and Germany, the contributions to this special issue highlight three points that should serve as guideposts for future research: the analysis of the interaction between education policy and federalism, studying the impact of the balance of power between political actors and the dynamics of political coalitions on reform processes, and the distinction between reform politics and policies on the one hand and processes of institutional change on the other.

\section{Introduction : \\ politique de la formation professionnelle et de l'enseignement supérieur en Suisse, Autriche et Allemagne}

Jusqu'à présent, la recherche en matière d'éducation sous l'angle spécifique de la science politique repose sur quatre piliers : l'étude comparative de la politique d'éducation ; le retracement analytique de l'internationalisation et l'européanisation des systèmes d'éducation; des ouvrages s'inscrivant dans la tradition de l'économie politique comparative ; l'analyse de l'effet généré par les politiques et institutions d'éducation. Sur la base d'une étude approfondie des transformations et réformes actuelles des systèmes d'éducation et de formation en Suisse, Autriche et Allemagne, les contributions 
de la présente édition spéciale mettent en évidence trois points spécifiques qui devront guider la recherche à l'avenir : l'étude de l'interaction entre la politique d'éducation et le contexte fédéral, l'étude de l'impact de la répartition du pouvoir entre acteurs politiques ainsi que la dynamique de certaines coalitions politiques sur le processus de réforme, et la distinction entre, d'un côté, la politique de réforme, et de l'autre côté les procès relatifs à la transformation institutionnelle.

Christine Trampusch ist Assistenzprofessorin für Vergleichende Politik am Institut für Politikwissenschaft der Universität Bern. Sie forscht, lehrt und publiziert in den Bereichen Vergleichende Politik, Qualitative Methoden, Europäisierung und Schweiz in Europa.

Korrespondenzadresse: Institut für Politikwissenschaft, Universität Bern, Unitobler, Lerchenweg 36, CH-3012 Bern, Schweiz. Tel.: +41 3163137 50; E-mail: christine.trampusch@ipw.unibe.ch.

Marius R. Busemeyer ist wissenschaftlicher Mitarbeiter (PD) am Max-Planck-Institut für Gesellschaftsforschung, Köln. Zum 1. Januar 2011 übernimmt er den Lehrstuhl für Politikwissenschaft an der Universität Konstanz. Seine Forschungsschwerpunkte sind die vergleichende politische Ökonomie von Bildung und Ausbildung, Wohlfahrtsstaaten, sozialdemokratische Parteien sowie öffentliche Finanzen und Haushaltspolitik.

Korrespondenzadresse: Max-Planck-Institut für Gesellschaftsforschung, Paulstrasse 3, D-50676 Köln, Deutschland. Tel.: +49 2212767 166; E-mail: busemeyer@mpifg.de. 